\title{
Characterization of the Tissue Distribution of the Mouse Cyp2c Subfamily by Quantitative PCR Analysis ${ }^{\mathrm{S}}$
}

\author{
Joan P. Graves, Artiom Gruzdev, J. Alyce Bradbury, Laura M. DeGraff, Matthew L. Edin, \\ and Darryl C. Zeldin
}

Immunity, Inflammation, and Disease Laboratory, National Institute of Environmental Health Sciences, National Institutes of Health, Research Triangle Park, North Carolina

Received February 28, 2017; accepted April 25, 2017

\begin{abstract}
The CYP2C subfamily of the cytochrome P450 gene superfamily encodes heme-thiolate proteins that have a myriad of biologic functions. CYP2C proteins detoxify xenobiotics and metabolize endogenous lipids such as arachidonic acid to bioactive eicosanoids. We report new methods and results for the quantitative polymerase reaction (qPCR) analysis for the 15 members of the mouse Cyp2c subfamily (Сyp2c29, Сyp2c37, Сyp2c38, Сyp2c39, Сур2c40, Сур2c44, Сур2c50, Сур2с54, Сур2с55, Сyp2c65, Сур2c66, Сур2с67, Сур2с68, Cyp2c69, and Cyp2c70). Commercially available TaqMan primer/probe assays were compared with developed SYBR Green primer sets for specificity toward the mouse Cyp2c cDNAs and analysis of their tissue distribution. TaqMan primer/probe assays for 10 of the mouse Cyp2c isoforms were shown to be specific for their intended mouse Cyp2c
\end{abstract}

\section{Introduction}

Members of the cytochromes P450 (P450s) gene superfamily have been intensely studied because of their ability to catalyze the metabolism of a broad array of xenobiotics and endogenous compounds (Nelson et al., 1996; Kroetz and Zeldin, 2002; Nebert and Russell, 2002). Arachidonic acid is a polyunsaturated fatty acid present in mammalian cell membranes that can undergo NADH-dependent oxidation by certain $\mathrm{P} 450$ s to generate bioactive metabolites such as epoxyeicosatrienoic acids (EETs) (Capdevila et al., 2000; Kroetz and Zeldin, 2002; Nebert and Russell, 2002). EETs have a wide range of physiologic functions including anti-inflammatory (Node et al., 1999), cardioprotective (Seubert et al., 2004, 2006), fibrinolytic (Node et al., 2001), angiogenic (Wang et al., 2005), and vasodilatory (Campbell et al., 1996; Larsen et al., 2006) effects both in vitro and in vivo.

One of the largest subfamilies of the P450s is the CYP2C subfamily. Many species contain multiple $C Y P 2 C$ subfamily members; rats have nine $C y p 2 c$ genes, whereas humans have four $C Y P 2 C$ genes (Nelson et al., 1996). The mouse Cyp2c subfamily is made up of 19 subfamily members, 4 of which are pseudogenes with no open reading frame. Eighteen of the nineteen genes are located within $\mathrm{a} \sim 1.5 \mathrm{Mb}$ cluster on

This work was supported by the Intramural Research Program of the National Institutes of Health National Institute of Environmental Health Sciences [Grant Z01 ES025034].

https://doi.org/10.1124/dmd.117.075697.

S This article has supplemental material available at dmd.aspetjournals.org.
cDNA; however, there were no TaqMan primer/probe assays specific for the mouse Cyp2c29, Cyp2c40, Cyp2c67, Cyp2c68, or Cyp2c69 transcripts. Each of the SYBR Green primer sets was specific for its intended mouse Cyp2c cDNA. The two qPCR methods confirmed similar patterns of Cyp2c tissue expression: Cyp2c37, Сyp2c38, Cyp2c39, Сyp2c44, Сyp2c50, Сyp2c54, and Cyp2c70 were most highly expressed in liver; Cyp2c55 was highly expressed in large intestine; Cyp2c65 was highly expressed in stomach, duodenum, and large intestine; and Cyp2c66 was highly expressed in both duodenum and jejunum. For isoforms without specific TaqMan primer/probe assays, the SYBR Green primer sets detected high level expression of Cyp2c29, Cyp2c40, Cyp2c67, Сyp2c68, and Cyp2c69 in the liver. Lower expression levels of the mouse Cyp2cs were also detected in other tissues.

mouse chromosome 19. The 19th gene, Cyp2c44, is located $\sim 3.8 \mathrm{Mb}$ away from the Cyp2c cluster (Nelson et al., 1996; Wang et al., 2004). Previous nonquantitative reverse-transcription polymerase chain reaction (RT-PCR) studies determined that most of the mouse Cyp2c subfamily members are highly expressed in the liver, but some are also expressed in extrahepatic tissues. Cyp2c29, Cyp2c37, Cyp2c38, Cyp2c39, Cyp2c40, Cyp2c50, and Cyp2c54 are all predominantly expressed in liver (Luo et al., 1998; Wang et al., 2004). Cyp2c55 is highly expressed in colon (Wang et al., 2004), whereas the Cyp $2 c 44$ epoxygenase has been shown to be expressed in liver and the renal collecting duct (DeLozier et al., 2004; Capdevila et al., 2014). The remaining mouse Cyp $2 c$ cDNAs (Cyp2c65, Cyp2c66, Cyp2c67, Cyp2c68, Cyp2c69, and Cyp2c70) were not previously cloned, and no analysis of their tissue distribution at the RNA level has been reported.

Although nonquantitative RT-PCR detects whether a particular transcript is present or not, it does not allow for the quantification of the transcript level. Quantitative polymerase chain reaction (qPCR) monitors the amplification of targeted sequences in real time, which allows accurate measurement of gene expression. For all qPCR reactions cDNA-specific primer pairs are used to amplify a target sequence from the transcript of interest. The SYBR Green qPCR method uses a doublestranded DNA-binding dye as the reporter (Ponchel et al., 2003). TaqMan or Quantifast qPCR methods rely on endonuclease activity cleaving a fluorophore from a transcript-specific probe containing a fluorescent quencher (Heid et al., 1996). Of the two methods, Taqman primer/probe assays have the advantage of increased selectivity due to their target-specific fluorescent probe. Unfortunately, researchers cannot

ABBREVIATIONS: EET, epoxyeicosatrienoic acid; qPCR, quantitative polymerase chain reaction; RT-PCR, reverse-transcription polymerase chain reaction. 
confirm whether the measured fluorescence of primer/probe assays is due to a single target amplicon as opposed to multiple off-target transcripts. This becomes especially important when quantifying members of a highly conserved subfamily of genes such as the mouse Cyp2cs, which have 53-98\% cDNA sequence identity (Table 1). DNAbinding assays such as SYBR Green might also fail to distinguish between highly related transcripts; however, they allow for performance of an end-point melting curve assay that can determine whether the observed fluorescence is due to a single amplicon or multiple unintended amplicons. Although the SYBR Green melting curve assay will not differentiate multiple amplicons of the same melting temperature, the end-point assay does provide a level of confirmation that is not possible with primer/probe qPCR assays.

In this study, SYBR Green primer sets were developed for use in qPCR assays based on published and cloned cDNA sequences of members of the mouse Cyp $2 c$ subfamily. These primer sets were compared with the commercially available mouse Cyp $2 c$ TaqMan primer/probe assays. Ten TaqMan primer/probe assays were shown to be specific for their intended transcript. All 15 of the SYBR Green primer sets were found to be specific for their intended transcript. Similar tissues distribution profiles were obtained using both qPCR methods.

\section{Materials and Methods}

Reagents and Supplies. SYBR Green primer set oligonucleotides were synthesized by Integrated DNA Technologies (Coralville, IA). TaqMan primer/ probes, MicroAmp Optical 384-Well Reaction Plates, an ABI Prism 7700 Sequence Detection System, and an ABI ViiA 7 Sequence Detection System were all purchased from Life Technologies (Carlsbad, CA).

RNA Isolation and cDNA Archives. Total RNA was prepared from three female and three male wild-type C57BL/6 mice for each tissue using RNeasy Midi or Mini Kits from Qiagen (Valencia, CA) following the manufacturer's protocols. For all the RNAs, $1 \mu \mathrm{g}$ of total RNA was treated with 1 unit of DNase I (Life Technologies) for 15 minutes and then inactivated by addition of $1 \mu \mathrm{l}$ $25 \mathrm{mM}$ EDTA and heat treatment at $65^{\circ} \mathrm{C}$ for 10 minutes. RNase-free water was then added to a total volume of $50 \mu 1$. The High Capacity cDNA Reverse Transcription Kit from Life Technologies was used according to the manufacturer's protocol to produce cDNA from the DNase I treated RNA. The final concentration of the cDNA archives was $10 \mathrm{ng} / \mu \mathrm{l}$, which was diluted to a working concentration of $1 \mathrm{ng} / \mu \mathrm{l}$ with RNase-free water.

Cloning of cDNAs for Cyp2c Subfamily Members. The cDNAs for Cyp2c65, Сyp2c67, Сyp2c68, and Cyp2c69 were identified by sequence analysis using the National Center for Biotechnology Information database's Basic Local Alignment Search Tool (BLAST) and the Lasergene DNAStar v12 package
(Madison, WI). Primer pairs were designed to amplify the coding regions of Cyp2c67, Cyp2c68, and Cyp2c69 cDNAs (Table 2). Primers for the Cyp2c65 cDNA included the $3^{\prime}$ untranslated region (UTR) sequence. Briefly, $60 \mathrm{ng}$ of stomach or liver cDNA archives (as described above) were amplified using Phusion HF DNA polymerase from New England BioLabs (Ipswich, MA) in a total volume of $25 \mu \mathrm{l}$. The PCR conditions were as follows: $96^{\circ} \mathrm{C}$ for 3 minutes, 35 cycles of $96^{\circ} \mathrm{C}$ for 10 seconds, $58^{\circ} \mathrm{C}$ for 10 seconds, and $72^{\circ} \mathrm{C}$ for 1 minute; the final extension was at $72^{\circ} \mathrm{C}$ for 2 minutes. The Cyp $2 c 68$ post-PCR reaction mix was digested with the restriction endonuclease enzymes NdeI and XmnI to eliminate any Cyp2c40, Cyp2c67, and Cyp2c69 amplicons. The PCR products were then gel isolated using a Qiagen kit. An A-overhang was added using New England BioLabs' Taq DNA polymerase, and the cDNA was cloned into the pRC2.1-TOPO vector from Life Technologies. Positive clones were identified using blue-white selection. Sequence fidelity was verified by DNA sequencing (GeneWiz, Inc., Research Triangle Park, NC).

TaqMan qPCR. Life Technologies' TaqMan primer/probe assays for the mouse Cyp $2 j$ c subfamily members are listed in Table 3 . For all qPCR reactions, $0.5 \mu \mathrm{l}$ of the $20 \times$ primer/probe assays and $5 \mu \mathrm{l}$ of Maxima Probe/ROX qPCR Master Mix $(2 \times)$ from Thermo Scientific (Pittsburgh, PA) were used in a $10 \mu \mathrm{l}$ total reaction volume, along with $1 \mathrm{ng}$ of the cDNA archive. The conditions used were $50^{\circ} \mathrm{C}, 2$ minutes; $95^{\circ} \mathrm{C}, 10$ minutes, followed by 40 repeat cycles of $95^{\circ} \mathrm{C}$, 15 seconds; $60^{\circ} \mathrm{C}, 1$ minute. Six individual samples for each tissue examined (three female and three male) were each run in triplicate ( 18 wells per tissue) on the same qPCR plate, except for sex-specific tissues where three individual tissue samples were each run in triplicate (nine wells per tissue) on the same plate.

SYBR Green qPCR. Specific primer sets of oligonucleotides were designed, spanning exon-exon boundaries when possible, for the protein coding members of the mouse Cyp $2 c$ subfamily (Table 4). Due to high sequence homology with other paralogs, the primer set for Cyp2c65 was designed in the $3^{\prime}$ UTR. Additional mouse Cyp2c primer sets that were used had previously been published for use in nonquantitative RT-PCR (Luo et al., 1998; Wang et al., 2004). All SYBR Green qPCR assays were run in $10 \mu \mathrm{l}$ volume with final primer concentration of $0.5 \mu \mathrm{M}$ for both the forward and reverse primers. Five microliters of Power SYBR Green PCR Master Mix (Applied Biosystems by Life Technologies) was used in the master mix for all the SYBR Green qPCR reactions, along with $1 \mathrm{ng}$ of cDNA archive. The conditions used were $50^{\circ} \mathrm{C}, 2$ minutes; $95^{\circ} \mathrm{C}, 10$ minutes, followed by 40 repeat cycles of $95^{\circ} \mathrm{C}, 15$ seconds; $60^{\circ} \mathrm{C}, 1$ minute (two-step PCR with single-stage annealing and extension). A melt curve dissociation stage was added of $95^{\circ} \mathrm{C}, 15$ seconds and then $60^{\circ} \mathrm{C}$ to $95^{\circ} \mathrm{C}$ at $0.05^{\circ} \mathrm{C} / \mathrm{s}$. For $C y p 2 c 29$, Cyp2c67, and Cyp2c68, an annealing/extension temperature of $68^{\circ} \mathrm{C}$ was used. For $C y p 2 c 39$ and $C y p 2 c 40$, an annealing/extension temperature of $64^{\circ} \mathrm{C}$ was used. For $C y p 2 c 54$, an annealing/extension temperature of $62^{\circ} \mathrm{C}$ was used. For Cyp $2 c 37$ and Cyp $2 c 69$ an annealing/extension temperature of $66^{\circ} \mathrm{C}$ was used. Six individual samples for each tissue examined (three female and three male) were each run in triplicate (18 wells per tissue) on the same qPCR plate, except

TABLE 1

Nucleic acid sequence identity of the mouse Cyp $2 c$ subfamily members

Full-length mouse Cyp2c cDNAs were compared using the Lipman-Pearson method in the DNAStar package (Madison, WI). Percent sequence identities are shown.

\begin{tabular}{|c|c|c|c|c|c|c|c|c|c|c|c|c|c|c|c|}
\hline & Сур $2 c 29$ & Сур $2 c 37$ & Сур $2 c 38$ & Сур $2 c 39$ & Сур $2 c 40$ & Cyp $2 c 44$ & Сур 2 c50 & Сур $2 c 54$ & Сур $2 c 55$ & Сур 2 c65 & Сур $2 c 66$ & Сур $2 c 67$ & Сур $2 c 68$ & Сур $2 c 69$ & Сур $2 c 70$ \\
\hline Сур $2 c 29$ & 100 & 75.8 & 86.9 & 87.9 & 69 & 55.4 & 77.3 & 71.9 & 64.6 & 70.6 & 69 & 67.4 & 68.3 & 68.9 & 62.6 \\
\hline Сур $2 c 37$ & & 100 & 72.8 & 88.3 & 72.6 & 63.9 & 95.8 & 81.9 & 64.8 & 73.1 & 68.2 & 73.3 & 71.6 & 72.8 & 60.7 \\
\hline Сур 2 c 38 & & & 100 & 89.5 & 67 & 56 & 77.1 & 70.5 & 62.6 & 73.8 & 75.4 & 70.2 & 68 & 65.9 & 58 \\
\hline Сур 2c39 & & & & 100 & 70.7 & 55.3 & 80.1 & 69 & 65.9 & 72.3 & 75.3 & 70.6 & 67.6 & 67.9 & 62.2 \\
\hline Сур $2 c 40$ & & & & & 100 & 59.1 & 74.4 & 70.4 & 58.5 & 69.7 & 66.9 & 98.7 & 97.9 & 97.2 & 57.2 \\
\hline Сур $2 c 44$ & & & & & & 100 & 58.2 & 53.2 & 56.2 & 65.3 & 62.2 & 54.3 & 52.8 & 56.1 & 60.2 \\
\hline Сур 2 c50 & & & & & & & 100 & 84.4 & 61.6 & 73.4 & 70.8 & 76.9 & 73.1 & 75.6 & 62.8 \\
\hline Сур $2 c 54$ & & & & & & & & 100 & 66.7 & 72.4 & 68.4 & 70.7 & 75.1 & 70.4 & 62.2 \\
\hline Сур $2 c 55$ & & & & & & & & & 100 & 74.2 & 70.6 & 65.1 & 64.8 & 65.9 & 63.8 \\
\hline Сур 2 c65 & & & & & & & & & & 100 & 98 & 66.4 & 64.3 & 64.7 & 62.7 \\
\hline Сур 2 c66 & & & & & & & & & & & 100 & 61.2 & 60.3 & 62.8 & 63.1 \\
\hline Сур 2 c67 & & & & & & & & & & & & 100 & 97.7 & 97.7 & 58.4 \\
\hline Сур 2 c68 & & & & & & & & & & & & & 100 & 96.1 & 57.5 \\
\hline Сур 2c69 & & & & & & & & & & & & & & 100 & 57.4 \\
\hline Сур $2 c 70$ & & & & & & & & & & & & & & & 100 \\
\hline
\end{tabular}


TABLE 2

Primer sequences used for the cloning of mouse Cyp2c65, Cyp2c67, Cyp2c68, and Cyp2c69 cDNAs

Corresponding positions are relative to the ATG initiating codon designated as +1 .

\begin{tabular}{|c|c|c|}
\hline cDNA and Direction & Primer Sequence $5^{\prime}$ to $3^{\prime}$ & Corresponding Position \\
\hline \multicolumn{3}{|l|}{ Cyp2c65 } \\
\hline Forward & GCAAACAATGGTTCTAGGTGTGTT & -6 to +17 \\
\hline Reverse & CCATCAGATAAGAACAGGGCTGA & +1807 to +1829 \\
\hline \multicolumn{3}{|c|}{ 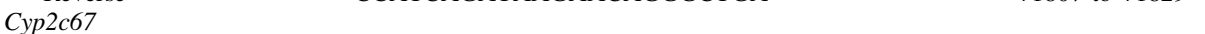 } \\
\hline Forward & GATCCATTTGTAGTCTTGGTGCTTTGTC & +4 to +31 \\
\hline Reverse & ATAGCAGAATCTTTCCTCATCTTCACTT & +1491 to +1518 \\
\hline \multicolumn{3}{|c|}{ 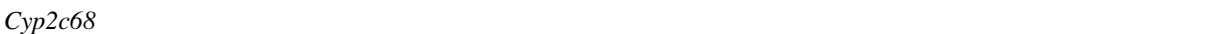 } \\
\hline Forward & ATCATACCAGTGTCAGTAAGAAGGG & -29 to -5 \\
\hline Reverse & ACTGTTAGCCTTGGGCAATTAGATA & +1605 to +1629 \\
\hline \multicolumn{3}{|l|}{ Сур 2 c69 } \\
\hline Forward & GAATGCCTGATCATAACAGTGTCAG & -38 to -14 \\
\hline Reverse & ATAACAGAGAACTTTCAGCCTTGGG & +1611 to +1635 \\
\hline
\end{tabular}

for sex-specific tissues where three individual tissue samples were each run in triplicate (nine wells per tissue) on the same plate.

qPCR Analysis. Data generated from qPCR was analyzed using the $2^{-\Delta \Delta C}$ method (Livak and Schmittgen, 2001). All graphs were generated using the GraphPad Prism 6.0C program (GraphPad Software, San Diego, CA). Mouse tissue expression values were normalized to glyceraldehyde 3-phosphate dehydrogenase (Gapdh) expression values. To confirm specificity against cloned cDNAs, SYBR Green qPCR assay values were normalized to $\beta$-lactamase (ampicillin resistance) from the cDNA vector. Outliers were determined using the Grubbs Outlier test (Grubbs, 1950). Significance for sex differences in various tissues was determined using the Student's $t$ test. No statistical comparisons were performed between tissues. The cDNA and tissue heat maps were generated using the Microsoft Excel program (Microsoft, Redmond, WA). Relative tissue expression was normalized to Gapdh; $\Delta \mathrm{CT}$ values (Cyp2c-Gapdh) are reported in the heat map (Fig. 2).

\section{Results}

Specificity of the Mouse Cyp2c TaqMan Primer/Probes and SYBR Green Primer Sets. The mouse Cyp $2 c$ subfamily cDNAs, except for Cyp2c65, Cyp2c67, Cyp2c68, and Cyp2c69, were previously cloned in collaboration with Dr. Joyce Goldstein's laboratory (Luo et al., 1998; DeLozier et al., 2004; Wang et al., 2004). Each cDNA was cloned into either the pBS vector (Agilent Technologies, Santa Clara, CA) or the pCR2.1 vector from Life Technologies. We cloned the Cyp2c65 cDNA from mouse stomach and the Cyp2c67, Cyp2c68, and Cyp2c69 cDNAs from mouse liver. The four new cDNAs were cloned into the pCR2.1 vector (ThermoFisher Scientific, Inc., Waltham, MA).

Ten of the eleven commercially available gene-specific mouse Cyp2c TaqMan primer/probe assays (Table 3) preferentially detected their target cDNAs (Fig. 1A). Only the Cyp2c29 probe failed to detect its respective cDNA and had cross-reactivity with the Cyp2c50, Cyp2c54, Cyp2c55, and Cyp2c66 cDNAs. The TaqMan Cyp2c29 probe amplifies an 89-bp amplicon completely located within terminal exon 9 . It may be that this amplicon spans both coding and noncoding sequence of exon 9. If this is the case, then the assay may be unable to detect the Cyp2c29 ORF clone, which lacks the complete $3^{\prime}$ UTR. The Cyp2c38 TaqMan primer/probe recognized the Cyp2c38 cDNA but also had some (30-35\%) cross-reactivity to the Cyp2c66 isoform (Supplemental Fig. 1A). Both the Cyp2c44 and the Cyp2c54 TaqMan primer/probes had low but detectable cross-reactivity to the Cyp2c39 and Cyp2c29 transcripts, respectively (Fig. 1A). Four TaqMan primer/probe assays (Table 3 ) were all advertised as having cross-reactivity between the Cyp2c40, Cyp2c67, Cyp2c68, and Cyp2c69 subfamily members. Results confirmed cross-reactivity of all four TaqMan primer/ probe assays for these four mouse Cyp2c isoforms (Fig. 1A).

The 15 mouse $C y p 2 c$ SYBR Green primer sets were specific for their intended ORF targets (Fig. 1B). The Cyp $2 c 29$ primer set had low but detectable cross-reactivity to the Cyp2c38 and Cyp2c50 cDNAs (Fig. 1B; Supplemental Fig. 1B), whereas the Cyp2c69 primer set had low but detectable cross-reactivity to the Cyp2c40, Cyp2c67, and Cyp2c68 isoforms (Fig. 1B).

The mouse Cyp $2 c$ and $C y p 2 j$ gene subfamilies are related with approximately $25-66 \%$ nucleotide identity (data not shown). To test for any cross-reactivity, the Cyp $2 c$ TaqMan primer/probes and SYBR Green primer sets were used in qPCR with the seven mouse $C y p 2 j$ cloned ORFs. None of the Cyp2c TaqMan primer/probes or SYBR Green primers sets detected any of the $C y p 2 j$ members (data not shown).

Tissue Distribution of the Mouse Cyp2c mRNAs by qPCR. By using the SYBR Green primer set, Cyp2c29 expression was most abundant in male liver with significantly lower expression in female liver; much lower expression was detected in duodenum and testis (Fig. 2B; Table 5; Supplemental Fig. 1D). Detection of Cyp2c29 expression in liver is consistent with published nonquantitative RT-PCR data, although no difference in expression between the sexes was previously reported (Luo et al., 1998). Cyp2c29 was also reported to be expressed in the intestines by nonquantitative RT-PCR (Luo et al., 1998), which is consistent with the expression of Cyp2c29 in duodenum by the SYBR Green primer set in the current work. Cyp2c29 was also detected in jejunum by the SYBR Green primers at low levels; however, the expression of Cyp $2 c 29$ in brain, kidney, lung, and heart by the nonquantitative RT-PCR method (Luo et al., 1998) was not confirmed using the SYBR Green qPCR method. There is an approximate melting temperature of $82.5^{\circ} \mathrm{C}$ for the dissociation curve of liver with the

TABLE 3.

TaqMan primer/probes for the mouse $C y p 2 c$ subfamily

\begin{tabular}{|c|c|}
\hline Subfamily Member & TaqMan Primer/Probe \\
\hline Cyp $2 c 29$ & Mm00725580_s1 \\
\hline Cyp $2 c 37$ & Mm00833845_m1 \\
\hline Cyp $2 c 38$ & Mm00658527_m1 \\
\hline Cyp 2 c39 & Mm04207909_g1 \\
\hline Сур 2c(40/67/69) & Mm04204172_mH \\
\hline Cyp $2 c 44$ & Mm01197188_m1 \\
\hline Cyp $2 c 50$ & Mm00663066_gH \\
\hline Cyp $2 c 54$ & Mm02602271_mH \\
\hline Cyp $2 c 55$ & Mm00472168_m1 \\
\hline Сур2c65 & Mm02344065_g1 \\
\hline Cyp $2 c 66$ & Mm01212038_g1 \\
\hline Cyp $2 c(67 / 69)$ & Mm03411955_mH \\
\hline Cyp $2 c(68 / 67 / 69)$ & Mm04204171_mH \\
\hline Сур $2 c(69 / 67 / 68)$ & Mm04204173_gH \\
\hline Cyp $2 c 70$ & Mm00521058_m1 \\
\hline Gapdh & Mm99999915_g1 \\
\hline
\end{tabular}


TABLE 4

SYBR Green primer set sequences for the mouse Cyp2c subfamily

\begin{tabular}{|c|c|c|c|c|}
\hline Primer & (Direction) & Sequence $\left(5^{\prime}-3^{\prime}\right)$ & Reference & Annealing/Extension Temperature \\
\hline \multirow[t]{2}{*}{ Cyp $2 c 29$} & Forward & GCTCTCCTACTCCTGCTGAAGT & & $68^{\circ} \mathrm{C}$ \\
\hline & Reverse & ATGTGGCTCCTGTCTTGCATGC & & \\
\hline \multirow[t]{2}{*}{ Cyp $2 c 37$} & Forward & CACGAGGCGTTTCTCACTCA & & $66^{\circ} \mathrm{C}$ \\
\hline & Reverse & AGGGCTGCTCAGAATCTTTGT & & \\
\hline \multirow[t]{2}{*}{ Cyp $2 c 38$} & Forward & CACTATGGAGACAGAGGTCTA & Luo et al., 1998 & $60^{\circ} \mathrm{C}$ \\
\hline & Reverse & CCAAATACAGAGTGAAAACG & & \\
\hline \multirow[t]{2}{*}{ Cyp $2 c 39$} & Forward & GGAGACAGAGCTGTGGC & Luo et al., 1998 & $64^{\circ} \mathrm{C}$ \\
\hline & Reverse & TAAAAACAATGCCAAGGCCG & & \\
\hline \multirow[t]{2}{*}{ Cyp $2 c 40$} & Forward & GGATTCCGGTTTTTGACAAGGTTTCTAC & & $64^{\circ} \mathrm{C}$ \\
\hline & Reverse & TGATTCTTCATGTTCTTTTATTTTCC & & \\
\hline \multirow[t]{2}{*}{ Cyp $2 c 44$} & Forward & GGCTATGATGTGGTGAAGGAAGC & & $60^{\circ} \mathrm{C}$ \\
\hline & Reverse & TCGCAGGAGTTTCCACCTCTCT & & \\
\hline \multirow[t]{2}{*}{ Cyp $2 c 50$} & Forward & GAAAACGGCAACTATCCAT & Wang et al., 2004 & $60^{\circ} \mathrm{C}$ \\
\hline & Reverse & CTTATGAAATTATGAGCAGGC & & \\
\hline \multirow[t]{2}{*}{ Cyp $2 c 54$} & Forward & CATGCCCTATACAAATGC & Wang et al., 2004 & $62^{\circ} \mathrm{C}$ \\
\hline & Reverse & GGCAGTGTGTTCAGTATGG & & \\
\hline \multirow[t]{2}{*}{ Cyp $2 c 55$} & Forward & AGGAAGCTCTGGATGACCTTGG & & $60^{\circ} \mathrm{C}$ \\
\hline & Reverse & TTGAGAAGCGCCGAAGCTCCTT & & \\
\hline \multirow[t]{2}{*}{ Cyp $2 c 65$} & Forward & TGTCCCAAGATGTTTCTTCACCA & & $60^{\circ} \mathrm{C}$ \\
\hline & Reverse & TTGCAGACTTGCTTTGCAGT & & \\
\hline \multirow[t]{2}{*}{ Cyp $2 c 66$} & Forward & GGAGTTTGCTGGAAGAGGAACC & & $60^{\circ} \mathrm{C}$ \\
\hline & Reverse & GCGTCTCATCTCTTTCCAGGTC & & \\
\hline \multirow[t]{2}{*}{ Cyp $2 c 67$} & Forward & GGAAGGTTTCCGTTTTTTGACAAGGTTA & & $68^{\circ} \mathrm{C}$ \\
\hline & Reverse & CCATGCCCAAGTTCCTCAAGGTATTGAT & & \\
\hline \multirow[t]{2}{*}{ Сур 2 c68 } & Forward & CTGTCCTTTCTGCTTCTCCTGTCAC & & $68^{\circ} \mathrm{C}$ \\
\hline & Reverse & AATACAGAGTGAACACAGGGCCATATA & & \\
\hline \multirow[t]{2}{*}{ Cyp $2 c 69$} & Forward & GGAGAAGTGTTCTCTGGAAGAGG & & $68^{\circ} \mathrm{C}$ \\
\hline & Reverse & GTGAAGACCCTTGTGGCTTTCC & & \\
\hline \multirow[t]{2}{*}{ Cyp $2 c 70$} & Forward & TGGGCTTTTGCTCCTGCTGAAG & & $60^{\circ} \mathrm{C}$ \\
\hline & Reverse & TCAGTGTACGGCATGTGGTTCC & & \\
\hline \multirow[t]{2}{*}{ Gapdh } & Forward & TTGATGGCAACAATCTCCAC & & $60^{\circ} \mathrm{C}$ \\
\hline & Reverse & CGTCCCGTAGACAAAATGGT & & \\
\hline \multirow[t]{2}{*}{$\beta$-Lactamase } & Forward & CGCAGAAGTGGTCCTGCAAC & & $60^{\circ} \mathrm{C}$ \\
\hline & Reverse & TCTGCTATGTGGCGCGGTAT & & \\
\hline
\end{tabular}

Cyp2c29 SYBR Green primer set (Supplemental Fig. 2A). The Cyp2c29 TaqMan primer/probe assay gave a similar tissue distribution pattern to that seen by the SYBR Green primer set (Fig. 2A; Supplemental Fig. 1C). It is interesting to note that Cyp2c29 is the only isoform with higher expression in male liver than in female liver.

Previous nonquantitative RT-PCR experiments, which used only female mouse tissues, demonstrated liver-specific expression of Cyp $2 c 37$ (Luo et al., 1998). Both qPCR methods also revealed Cyp2c37 expression was highest in liver, but with significantly greater expression in female liver than in male liver (Fig. 2; Table 5; Supplemental Fig. 3). With liver, a single dominant peak for the dissociation curve with the Cyp2c37 SYBR Green primers had a melting temperature of approximately $82^{\circ} \mathrm{C}$ (Supplemental Fig. 2B).

Prominent Cyp 2 c 38 transcripts were found in both male and female livers with a significantly higher expression in female versus male liver using both qPCR methods (Fig. 2; Table 5; Supplemental Fig. 4). Much lower transcript levels were detected with the Cyp2c38 TaqMan qPCR assay in duodenum, jejunum, kidney, lymph node, skin, testis, and uterus, whereas the SYBR Green primer set detected lower transcript levels only in duodenum and ovary. For the SYBR Green Cyp $2 c 38$ primer set, the dissociation curve for liver was found to have a single dominant peak with a melting temperature of approximately $78.5^{\circ} \mathrm{C}$ (Supplemental Fig. 2C). The expression detected in duodenum, jejunum, kidney, lymph node, skin, testis, and uterus by the Cyp2c38 TaqMan primer/probe may be explained by its crossreactivity to Cyp2c66, which is highly expressed in the gastrointestinal tract and testis (Fig. 2; Supplemental Fig. 12). Nonquantitative RT-PCR, using only female mouse tissues, previously demonstrated the presence of the Cyp $2 c 38$ transcript in liver, brain, kidney, and intestine with much lower levels of expression in lung and heart (Luo et al., 1998).

Published nonquantitative RT-PCR experiments using only female mouse tissues demonstrated Cyp2c39 expression specifically in liver (Luo et al., 1998). qPCR results from the Cyp2c39 TaqMan primer/ probe and SYBR Green primer set confirmed highest expression in the liver, but also revealed higher Cyp2c39 expression in female liver than in male liver (Fig. 2; Table 5; Supplemental Fig. 5). Cyp2c39 was also found at much lower but detectable levels in other tissues examined, including aorta, kidney, large intestine, and lung, with both qPCR methods. The dissociation curve for liver with the SYBR Green Cyp2c39 primer set revealed a single prominent peak with a melting temperature of $80.3^{\circ} \mathrm{C}$ (Supplemental Fig. 2D).

For Cyp2c40, the highest expression using the SYBR Green primer set was in the liver. Cyp2c40 is more highly expressed in female liver compared with male liver (Fig. 2; Table 5; Supplemental Fig. 6). A single dominant peak was obtained only for liver with an approximate melting temperature of $80.5^{\circ} \mathrm{C}$ (Supplemental Fig. 2E). In contrast, previous nonquantitative RT-PCR experiments detected Cyp $2 c 40$ in liver, intestine, brain, and kidney with lower expression also present in lung and heart (Luo et al., 1998). There is no Cyp2c40-specific TaqMan primer/probe available. All four TaqMan primer/probes tested (Table 3) that recognized the Cyp2c40 cDNA also recognized Cyp2c67, Cyp2c68, and Cyp2c69 (Fig. 1; Supplemental Figs. 6A, 13A, 14A, and 15A). Therefore, although a similar expression pattern to the Cyp2c40 SYBR Green primer assays was obtained with these four TaqMan primer/ probes, none can be used as a Cyp $2 c 40$-specific primer/probe assay.

By nonquantitative RT-PCR methods, the Cyp2c44 transcript was previously reported to be expressed in liver, kidney, and adrenal gland; 
A
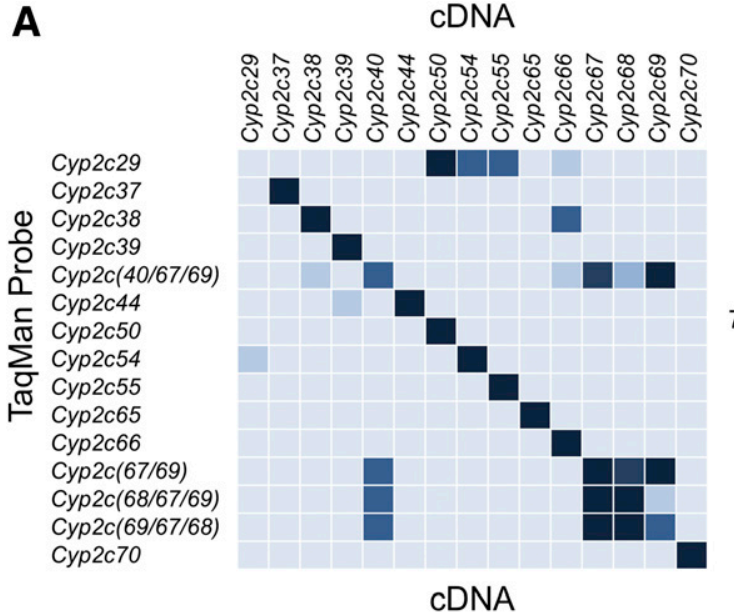

B
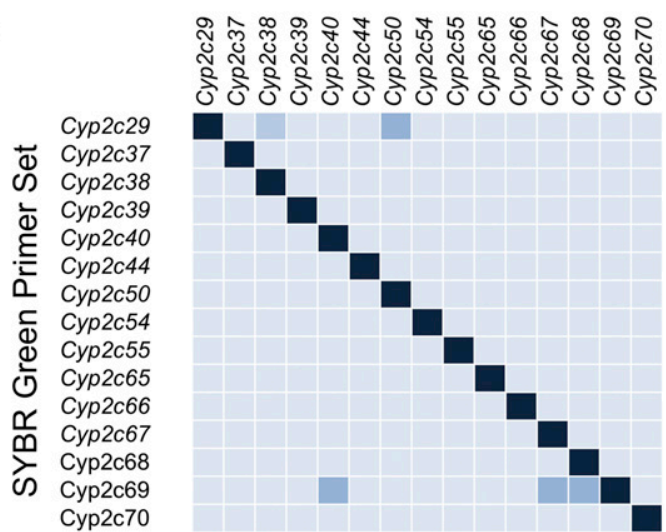

Fig. 1. Heat maps showing specificity of TaqMan primer/probes and SYBR Green primer sets for mouse Cyp2c cDNAs. (A) Both the Cyp2c29 and Cyp2c38 TaqMan primer/probes are cross-reactive with other $C y p 2 c$ genes. The probes used for Cyp2c40, Cyp2c67, Cyp2c68, and Cyp2c69 comparisons are nonspecific. (B) All the mouse $C y p 2 c$ SYBR Green primer sets are specific to their intended $C y p 2 c$ gene target.

Cyp2c44 was found to be similarly expressed in male and female livers, but more highly expressed in female kidney and adrenal gland compared with male (DeLozier et al., 2004). By both qPCR methods, Cyp $2 c 44$ transcripts were detected at approximately equal levels in male and female livers (Fig. 2; Table 5; Supplemental Fig. 7). Cyp $2 c 44$ was also detected in kidney, with significantly higher expression in female kidney compared with male kidney. Both methods confirm the previous nonquantitative RT-PCR results. Adrenal glands were not included in the panel of tissues for the current study. Low levels of Cyp2c44 expression were observed in several other tissues examined. Both qPCR methods detected Cyp $2 c 44$ in liver, duodenum, kidney, testis, stomach, lung, ileum, jejunum, large intestine, esophagus, and visceral fat. The SYBR Green dissociation curves yielded a single peak for all these tissues. The dissociation curve for liver with the Cyp2c44 SYBR Green primer set gives a melting temperature of $79.5^{\circ} \mathrm{C}$, as shown in Supplemental Fig. 2F. This finding stands in contrast to the earlier nonquantitative results, which found no detectable expression level in other tissues including small intestine, colon, testis, and lung.

A prominent Cyp 2 c50 transcript was detected in liver samples using both TaqMan and SYBR Green qPCR methods (Fig. 2; Table 5; Supplemental Fig. 8). Expression levels for Cyp2c50 were approximately the same for both male and female liver using both qCPR methods. For the SYBR Green primer set, very low levels of Cyp $2 c 50$ transcript (CT values of $\sim 36$ ) were detected in the remaining tissues tested. The SYBR Green Cyp2c50 dissociation curves were examined and only liver gave a single dominant peak with a melting temperature of approximately $81.5^{\circ} \mathrm{C}$ (Supplemental Fig. $2 \mathrm{G}$ ), which suggests that the low level of detection in other tissues represents cross-reactivity with nontarget transcripts. Previous nonquantitative RT-PCR methods detected the expression of Cyp2c50 in the liver and heart (Wang et al., 2004). Heart expression of Cyp2c50 was not confirmed by either qPCR method used in this study.

Prominent Cyp2c54 expression was detected in male and female liver using both qPCR methods. Significantly higher expression was detected in female liver compared with male liver (Fig. 2; Table 5; Supplemental Fig. 9). There was low level detection of Cyp2c54 in all other tissues examined with the SYBR Green primer set. For the Cyp2c54 SYBR Green dissociation curves, only liver produced a single prominent peak with a melting temperature of approximately $81.5^{\circ} \mathrm{C}$ (Supplemental Fig. $2 \mathrm{H})$. A single peak was observed only for liver. The low level detection in all other tissues is most likely due to cross-reactivity with nontarget transcripts. Nonquantitative RT-PCR from previous studies reported Cyp2c54 transcripts in liver, stomach, and kidney (Wang et al., 2004). Our methods can only confirm significant expression of Cyp2c54 in liver.

Previous findings with nonquantitative RT-PCR (Wang et al., 2004) demonstrated that Cyp2c55 transcripts were present in all the tissues examined (liver, kidney, lung, heart, brain, spleen, colon, small intestine, stomach, testis, skeletal muscle, aorta, adrenal gland, prostate, and ovary). By using both the TaqMan primer/probe and SYBR Green primer sets, Cyp2c55 was found to be most abundant in the large intestine with significantly higher expression in male versus female large intestine (Fig. 2; Table 5; Supplemental Fig. 10). Lower Cyp2c55 expression was found in the jejunum with significantly higher expression in male jejunum versus female jejunum. Low levels of Cyp2c55 expression were also found in duodenum, ileum, stomach, and liver. The other tissues failed to give a single peak in the Cyp $2 c 55$ SYBR Green dissociation curves. The single dominant peak for large intestine has an approximate melting temperature of $81.5^{\circ} \mathrm{C}$ (Supplemental Fig. 2I). This suggests off-target cross-reactivity may account for previous nonquantitative RT-PCR results.

The Cyp2c65 SYBR Green primer set amplifies a region of the $3^{\prime}$ UTR. The Cyp2c65 SYBR Green dissociation curves gave a single prominent peak with an approximate melting temperature of $76.5^{\circ} \mathrm{C}$ for stomach, duodenum, ileum, jejunum, large intestine, testis, and esophagus (Fig. 3; Supplemental Fig. 2J). To confirm specificity for Cyp2c65, the SYBR Green qPCR reactions for stomach, duodenum, and large intestine were run on agarose gels. A single band was obtained for each, which was isolated using a Qiagen Gel Isolation Kit. The isolated bands were sequenced using the Cyp2c65 SYBR Green primers. For all three tissues tested, the sequence matched the predicted Cyp2c65 sequence (Fig. 3B). This strongly suggests that the SYBR Green Cyp $2 c 65$ primer set specifically detect only the Cyp $2 c 65$ transcript in these tissues. Cyp2c65 transcripts were detected in stomach, duodenum, and large intestine with much lower expression in jejunum, testis, and esophagus by both qPCR methods (Fig. 2; Table 5; Supplemental Fig. 11). There was significantly higher expression in female large intestine than male large intestine by both qPCR methods. There was also significantly higher expression in male jejunum compared with female jejunum with the Cyp2c65 TaqMan primer/probe. This sex difference in the SYBR Green primer assay was not statistically significant $(P=0.07)$. No previous RT-PCR studies have been performed for Cyp2c65.

Prominent Cyp2c66 transcripts were detected in duodenum and jejunum, with significantly lower expression in large intestine and testis using both qPCR methods (Fig. 2; Table 5; Supplemental Fig. 12). There 


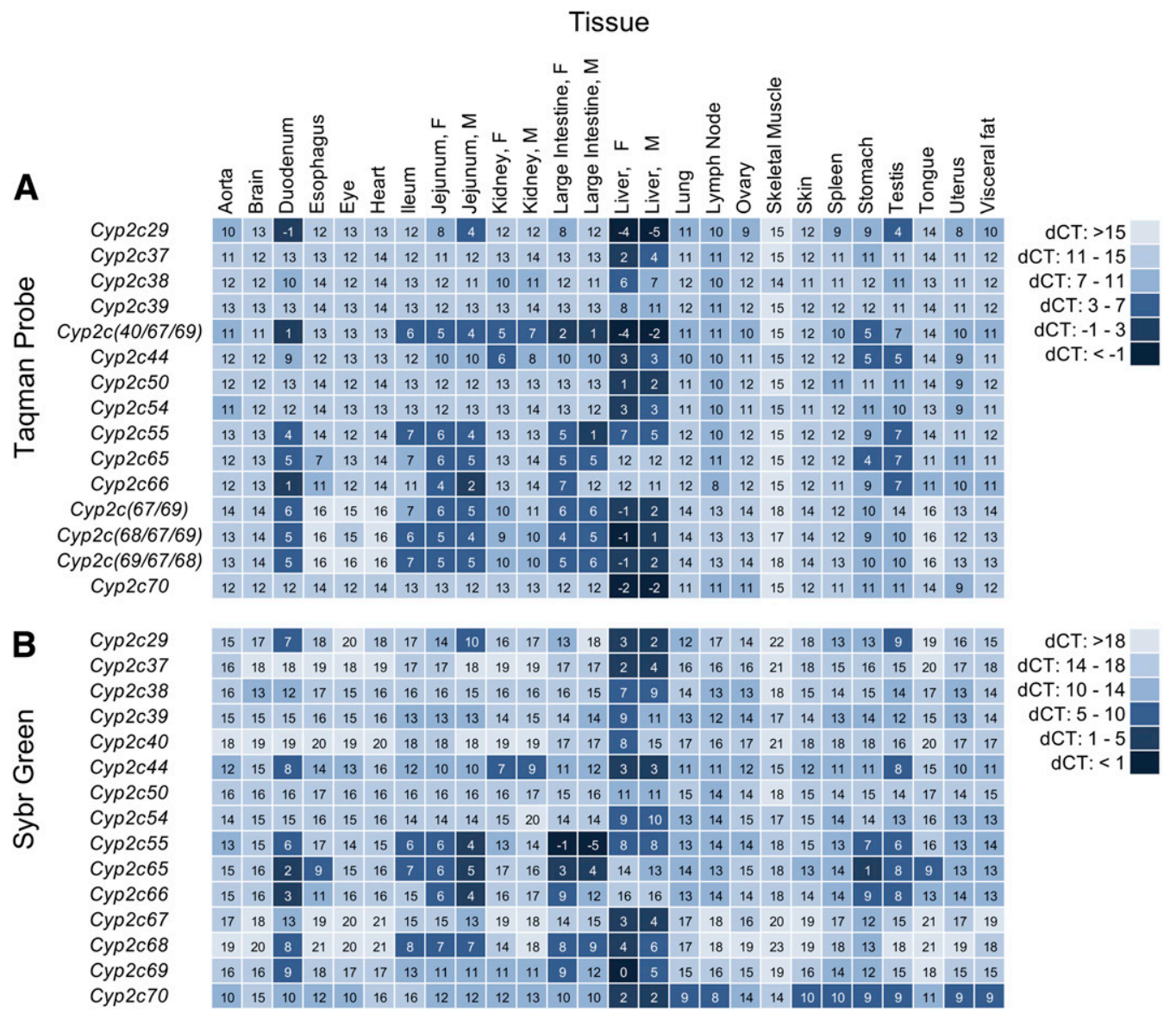

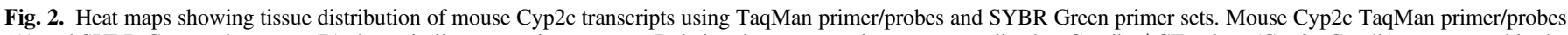

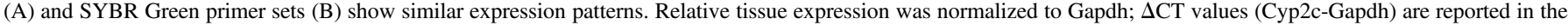
heat maps.

was significantly higher expression in male jejunum than female jejunum. Conversely, there was significantly higher expression in female large intestine compared with male large intestine using both qPCR methods. A single dominant peak for duodenum and jejunum gave an approximate melting temperature of $79.0^{\circ} \mathrm{C}$ for the Cyp $2 c 66$ SYBR Green primer set (Supplemental Fig. 2K). Cyp2c66 expression has not previously been reported.

There were no previous nonquantitative RT-PCR studies found with mouse $C y p 2 c 67$. We detected a prominent $C y p 2 c 67$ transcript in liver using the SYBR Green primer set with similar expression in both sexes (Fig. 2; Table 5; Supplemental Fig. 13D). There is no Cyp2c67specific TaqMan primer/probe assay available (Fig. 1). The TaqMan primer/probe (Table 3) used for comparison with the Cyp2c67 SYBR Green primers also strongly recognizes the Cyp $2 c 40$, Cyp $2 c 68$, and Cyp2c69 transcripts (Supplemental Fig. 13A). This cross-reactivity likely accounts for the higher expression observed in female liver in comparison with male liver, because this sex difference is also seen using the Cyp2c40-, Cyp2c68-, and Cyp2c69-specific SYBR Green primer sets (Supplemental Figs. 6D, 14D, and 15D). A single prominent peak was observed using the SYBR Green primers with liver that had an approximate melting temperature of $77.5^{\circ} \mathrm{C}$ (Supplemental Fig. 2L).

The Cyp2c68 SYBR Green primer set detects the highest expression in liver with significantly greater expression in female liver versus male liver (Fig. 2; Table 5; Supplemental Fig. 14). Cyp2c68 transcripts were also detected in duodenum, ileum, jejunum, large intestine, stomach, and kidney, which is confirmed by the dissociation curves. A single dominant peak for liver has an approximate melting temperature of $80.0^{\circ} \mathrm{C}$ (Supplemental Fig. 2M). There was significantly higher expression of Cyp2c68 in female large intestine compared with male large intestine. There is no specific Cyp2c68 TaqMan primer/probe available (Fig. 1; Supplemental Fig. 14A). The expression pattern seen with the TaqMan primer/probe used for comparison (Table 3 ) could be due to detection of Cyp2c40, Cyp2c67, and Cyp2c69, as well as Cyp $2 c 68$, because all four isoforms are recognized by this primer/probe assay. No nonquantitative RT-PCR experiments for Cyp2c68 expression were previously reported.

A prominent transcript is detected in liver using the Cyp2c69specific SYBR Green primers (Fig. 2; Table 5; Supplemental Fig. 15). There is significantly higher Cyp2c69 expression in female liver compared with male liver. The dissociation curves for the Cyp2c69 SYBR Green primer set reveals a single peak with a melting temperature of approximately $77.0^{\circ} \mathrm{C}$ (Supplemental Fig. $2 \mathrm{~N}$ ). There is no specific TaqMan primer/probe assay for mouse Cyp2c69. The Cyp2c69 TaqMan primer/probe assay (Table 3) used also recognizes Cyp2c40, Cyp2c67, and Cyp2c68 (Supplemental Fig. 15A). The expression pattern observed with this primer/probe could be due to reactivity with any of the other four isoforms (Fig. 1). No nonquantitative RT-PCR experiments for mouse Cyp2c69 were available as a comparison.

Finally, both qPCR methods detected Cyp2c70 only in liver (Fig. 2; Table 5; Supplemental Fig. 16). There were no expression differences between the sexes. The dissociation curves for liver gave a single dominant peak with an approximate melting temperature of $79.5^{\circ} \mathrm{C}$ 
TABLE 5

Mouse Cyp2c subfamily expression summary

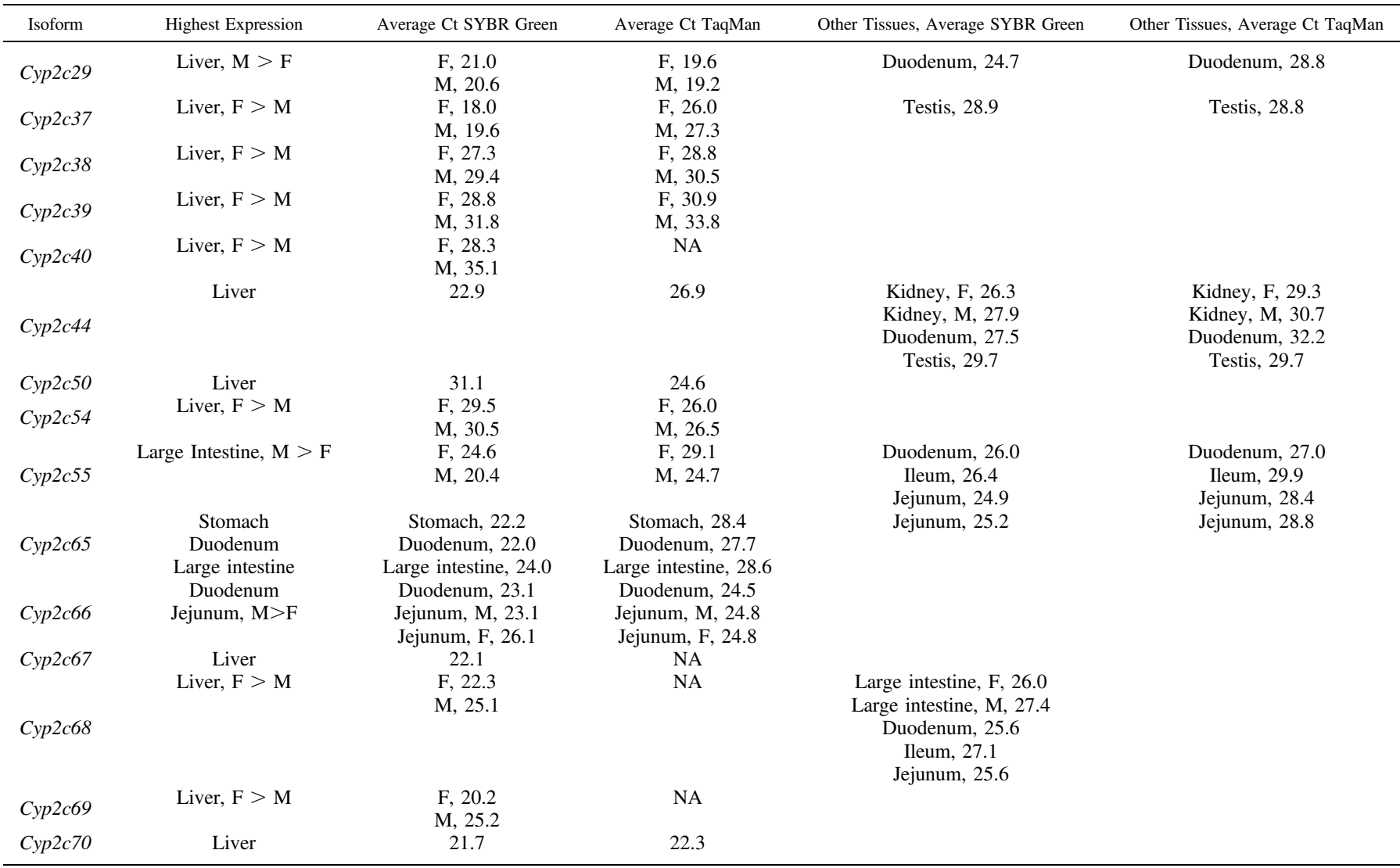

NA, Specific probes not available.

(Supplemental Fig. 2O). No previous Cyp $2 c 70$ nonquantitative RT-PCR experiments have been reported.

\section{Discussion}

The four human $C Y P 2 C$ isoforms $(C Y P 2 C 8, C Y P 2 C 9, C Y P 2 C 18$, and $C Y P 2 C 19)$ account for $20 \%$ of hepatic P450s and are active in the metabolism of many widely used drugs (Goldstein and de Morais, 1994) and endogenous compounds (Daikh et al., 1994; Zeldin et al., 1995). In comparison, the mouse Cyp $2 c$ subfamily is larger and more complex, with 15 subfamily members (Nelson et al., 1996; Wang et al., 2004). Mouse Cyp $2 c$ enzymes also metabolize both endogenous and exogenous compounds (Luo et al., 1998; DeLozier et al., 2004; Wang et al., 2004). They are predominantly expressed in liver but are also present in extrahepatic tissues (Luo et al., 1998; DeLozier et al., 2004; Wang et al., 2004). Accurate identification of the tissue expression pattern of the mouse Cyp $2 c$ isoforms is a critical step in identifying their physiologic roles. The tissue distribution of nine of the fifteen members of the mouse Cyp $2 c$ subfamily had been previously reported using both nonquantitative RT-PCR and immunoblotting (Luo et al., 1998; DeLozier et al., 2004; Wang et al., 2004). Analysis of the mouse Cyp2c subfamily members in a panel of tissues by qPCR provides a more reliable tissue expression profile. Herein, we describe 1) development of new SYBR Green primer sets, 2) specificity of both TaqMan and SYBR Green qPCR methods for each of the 15 mouse Cyp $2 c$ isoforms, 3) tissue expression patterns for all 15 mouse Cyp2c subfamily members using our developed SYBR Green primer sets, and 4) tissue expression patterns of 11 of the mouse Cyp2c isoforms using specific TaqMan primer/probes. In short, our work provides a valuable reference for mouse $C y p 2 c$ qPCR methods and catalogs their tissue distribution.

Nine of the fifteen mouse TaqMan primer/probes tested (Cyp2c37, Cyp2c39, Сyp2c44, Сyp2c50, Cyp2c54, Сyp2c55, Сyp2c65, Сур2c66, and $C y p 2 c 70$ ) are highly specific for their respective $C y p 2 c$ isoforms. The Cyp2c38 TaqMan primer/probe recognizes its intended cDNA, but has $\sim 30-35 \%$ cross-reactivity to the Cyp $2 c 66$ cDNA. This crossreactivity is not surprising due to the $\sim 75 \%$ identity between the Cyp2c38 and Cyp2c66 cDNAs. The Cyp2c29 TaqMan primer/probe does not recognize its intended cDNA, but this is most likely due to the probe partially targeting the 3' UTR that is absent from our cDNA. The remaining TaqMan primer/probes used for this study are not advertised as being specific for the Cyp2c40, Cyp2c67, Cyp2c68, and Cyp2c69 isoforms. These assays were included for comparison with the SYBR Green primer sets developed for these Cyp $2 c$ subfamily members.

The 15 mouse $C y p 2 c$ SYBR Green primer sets are highly specific for their intended Cyp2c cDNA. These include the four isoforms (Cyp2c40, Cyp2c67, Cyp2c68, and Cyp2c69) for which there are no specific TaqMan primer/probes.

Tissue expression profiles of the mouse Cyp $2 c$ isoforms were generally similar using both qPCR methods. Of the 15 mouse Cyp2c subfamily members, 12 were most highly expressed in liver. Mammalian P450s highly expressed in liver have important roles in the metabolism of certain drugs (Guengerich, 1992). Human Cyp2cs are known to metabolize clinically relevant drugs such as tolbutamide, which is used for the management of type 2 diabetes (Veronese et al., 1991), as well as anti-inflammatory drugs such as ibuprofen and acetylsalicylic acid (aspirin) (Leemann et al., 1993). The mouse 
A

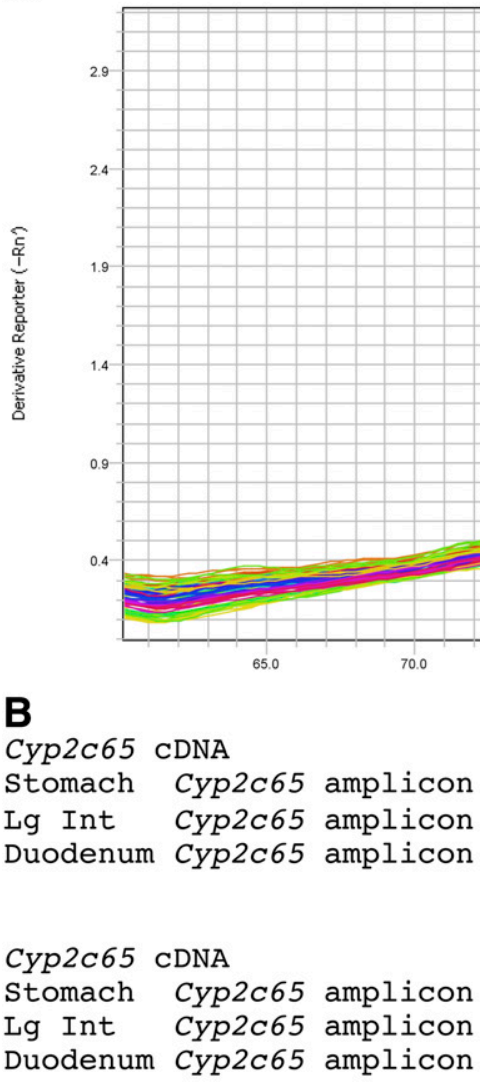

Melt Curve Plot
Fig. 3. Cyp2c65 SYBR Green primer set dissociation curves and sequence. (A) A single peak in the Cyp2c65 melting curve plot was detected for duodenum, ileum, jejunum, large intestine, stomach, testis, and esophagus. (B) Sequencing of the single band gel-isolated from the stomach, large intestine, and duodenum SYBR Green qPCR reactions gave the expected Cyp2c65 cDNA sequence. The primer sequences are indicated by capital letters.

\section{aggaagcatgacatctctggccatctatctcttaa tctctggccatctatctcttaa
tctctggccatctatctcttaa
tctctggcnntctatctcttaa tctctggccatctatctcttaa
tctctggccatctatctcttaa
tctctggcnntctatctcttaa tctctggccatctatctcttaa
tctctggccatctatctcttaa
tctctggcnntctatctcttaa} 71 105 agatacaggaaatgcctatcttctatttaaggcaa agatacaggaaatgcctatctctatttaaggcaa agatacaggaaatgcctatcttctatttaaggcaa agatacaggaaatgcctatcttctatttaaggcaa

106

ttattagggttACTGCAAAGCAAGTCTGCAA

ttattagggttactgcaaagcaagtctgcaa

ttattagggttactgcaaagcaagtctgcaa

ttattagggttactgcaaagcaagtctgcaa
Stomach Cyp2c65 amplicon Lg Int Cyp2c65 amplicon Duodenum Cyp2c65 amplicon

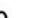

(1)

Cyp2c29, Cyp2c38, and Cyp2c39 isoforms have been shown to metabolize tolbutamide (DeLozier et al., 2004). Mouse Cyp2c29 and Cyp $2 c 37$ are inducible by phenytoin and the Cyp $2 c 29$, Cyp $2 c 37$, and Cyp 2c55 isoforms are inducible by phenobarbital (Jackson et al., 2004, 2006; Konno et al., 2010). It will be important to test the remaining mouse Cyp2cs for their ability to metabolize drugs to better understand their physiologic roles in the liver.

Six of the twelve mouse $C y p 2 c$ enzymes that are most highly expressed in the liver (Cyp2c37, Cyp2c38, Cyp2c39, Cyp2c40, Cyp2c54, and Cyp2c69) are found to have significantly higher expression in female liver compared with male liver. There is only one isoform (Cyp2c29) that has significantly higher expression in male liver compared with female liver. It is well known that sex differences found in the metabolism of many pharmaceuticals are due, at least in part, to sex differences in expression of hepatic enzymes, including P450s (Waxman and Azaroff, 1992; Waxman and Holloway, 2009). Identifying these sexually dimorphic mouse Cyp $2 c$ enzymes in liver may aid in understanding differences in drug potency observed in male versus female mice. However, sexual dimorphism is much less pronounced in human models in comparison with rodent models (Lofgren et al., 2008; Tanaka, 1999), which must be taken into account when using the mouse as a model for human disease and drug metabolism.

Cytochrome $\mathrm{P} 450$ enzymes primarily expressed in the gastrointestinal tract may also play an important role in drug elimination as well as biotransformation of other exogenous and endogenous compounds (Kaminsky and Fasco, 1992; Wang et al., 2004; Paine et al., 2006). Both qPCR methods show that Cyp2c55, Cyp2c65, and Cyp2c66 are highly expressed in the gastrointestinal tract with similar tissue expression profiles for each isoform. Previously Cyp $2 c 40$ was identified as the major $C y p 2 c$ isoform present in the intestine (Tsao et al., 2000); however, with more sensitive and paralog-specific qPCR methods, we identified other highly expressed $C y p 2 c$ isoforms in the intestinal tract and that $C y p 2 c 40$ is predominantly expressed in female liver.

Mouse models exist that have disruption of Cyp2c isoforms. Cyp $2 c 44^{-1-}$ mice have hyperactive epithelial sodium channel (ENaC) activity in the kidney and are more susceptible to salt-induced hypertension than wild-type mice. The authors concluded that 
CYP2C44 normally generates EETs to suppress ENaC activity and limit sensitivity to high-salt diet (Capdevila et al., 2014). Our data suggest that Cyp $2 c 44$ is the predominant Cyp $2 c$ expressed in the kidney. Another mouse model has the 14 Cyp $2 c$ gene cluster genetically disrupted with only the downstream Cyp $2 c 44$ locus intact. These mice are viable and healthy, although some animals exhibited liver inflammation. They also have significantly reduced metabolism of known CYP2C substrates such as tolbutamide (Scheer et al., 2012). Together, these models will be important for elucidating the roles of mouse CYPC enzymes in cardiovascular physiology and xenobiotic metabolism. The human ortholog of mouse Cyp $2 c 44$ is not known. Human CYP2C8 and CYP2C9, thought to be the primary CYP2C epoxygenases, are both abundantly expressed in kidney and have a similar function (Enayetallah et al., 2004). Studies with the human single nucleotide polymorphism CYP2C8*3 have shown either a small but significant association with hypertension (Tzveova et al., 2015) or no association with hypertension (Dreisbach et al., 2005; King et al., 2005). Further studies will be needed to clarify the association between polymorphisms such as CYP2C8*3 and hypertension in humans.

Although the data in this manuscript provide a comprehensive profile of $C y p 2 c$ gene expression in mice, they do little to clarify the identity of the murine orthologs for the known human $C Y P 2 C$ genes. Humans have four $C Y P 2 C$ genes, $C Y P 2 C 8, C Y P 2 C 9, C Y P 2 C 18$, and $C Y P 2 C 19$ (Nelson et al., 2004). Collectively, we find murine Cyp $2 c$ expression is restricted largely to the liver, gastrointestinal tract, and kidney. In humans, $C Y P 2 C 8$ and $C Y P 2 C 9$ are expressed in all three of these tissues, but can also be found in other tissues, including heart, lung, lymph nodes, spleen, and skin (Enayetallah et al., 2004). Metabolic studies with individual CYP2C proteins will be needed to clarify whether any of the murine CYP2Cs perform similar functions as the corresponding human CYP2C enzymes. Due to the complexity and high level of homology between the mouse Cyp2c subfamily members, it is exceedingly difficult to develop isoform-specific antibodies. Future Western blot analysis and immunohistochemistry studies will be essential to confirm the tissue distribution and sexual dimorphism for the mouse Cyp2c proteins. In addition, although human CYP2Cs also display sexually dimorphic expression, this occurs to a lesser degree than in mice. It is important to note these differences to fully understand and interpret drug metabolism data by CYP2Cs in humans compared with mice.

In summary, we developed SYBR Green primers, validated their specificity, and compared them to commercially available Taqman primer/probe assays for determination of the tissue expression profiles of the 15 mouse Cyp $2 c$ subfamily members. Similar tissue expression profiles were observed for 11 of the mouse $C y p 2 c$ isoforms using both qPCR methods. Tissue expression profiles were also developed using our SYBR Green primer sets for the remaining four mouse Cyp $2 c$ isoforms that currently do not have paralog-specific TaqMan primer/ probe assays. Although primer/probe assays may offer increased specificity of target detection, the SYBR Green end-point melting curve dissociation analysis allows for verification that observed fluorescence is due to a single amplicon (or at least amplicons of similar melting temperature). Our developed mouse Cyp2c SYBR Green primer sets may be used as accurate and less expensive alternatives to the commercially available TaqMan primer/probe assays for the quantitative expression of the mouse Cyp2cs transcripts in tissues. In addition, our Cyp $2 c$-specific SYBR Green primer sets may be used for the quantitative analysis in tissues where there is no specific TaqMan assay available.

\section{Acknowledgments}

The authors are grateful to Lois Wyrick for graphic arts assistance.

\section{Authorship Contributions}

Participated in research design: Graves, Gruzdev, Edin, and Zeldin. Conducted experiments: Graves.

Contributed new reagents or analytical tools: Bradbury and DeGraff.

Performed data analysis: Graves, Gruzdev, and Zeldin.

Wrote or contributed to the writing of the manuscript: Graves, Gruzdev, Edin, and Zeldin.

\section{References}

Campbell WB, Gebremedhin D, Pratt PF, and Harder DR (1996) Identification of epoxyeicosatrienoic acids as endothelium-derived hyperpolarizing factors. Circ Res 78:415-423.

Capdevila JH, Falck JR, and Harris RC (2000) Cytochrome P450 and arachidonic acid bioactivation. Molecular and functional properties of the arachidonate monooxygenase. J Lipid Res 41:163-181.

Capdevila JH, Pidkovka N, Mei S, Gong Y, Falck JR, Imig JD, Harris RC, and Wang W (2014) The Cyp2c44 epoxygenase regulates epithelial sodium channel activity and the blood pressure responses to increased dietary salt. J Biol Chem 289:4377-4386.

Daikh BE, Lasker JM, Raucy JL, and Koop DR (1994) Regio- and stereoselective epoxidation of arachidonic acid by human cytochromes P450 2C8 and 2C9. J Pharmacol Exp Ther 271: $1427-1433$.

DeLozier TC, Tsao CC, Coulter SJ, Foley J, Bradbury JA, Zeldin DC, and Goldstein JA (2004) CYP2C44, a new murine CYP2C that metabolizes arachidonic acid to unique stereospecific products. J Pharmacol Exp Ther 310:845-854.

Dreisbach AW, Japa S, Sigel A, Parenti MB, Hess AE, Srinouanprachanh SL, Rettie AE, Kim H, Farin FM, Hamm LL, et al. (2005) The Prevalence of CYP2C8, 2C9, 2J2, and soluble epoxide hydrolase polymorphisms in African Americans with hypertension. Am J Hypertens 18: $1276-1281$.

Enayetallah AE, French RA, Thibodeau MS, and Grant DF (2004) Distribution of soluble epoxide hydrolase and of cytochrome $\mathrm{P} 4502 \mathrm{C} 8,2 \mathrm{C} 9$, and $2 \mathrm{~J} 2$ in human tissues. J Histochem Cytochem 52:447-454.

Goldstein JA and de Morais SM (1994) Biochemistry and molecular biology of the human CYP2C subfamily. Pharmacogenetics 4:285-299.

Grubbs FE (1950) Sample criteria for testing outlying observations. Ann Math Stat 21:27-58.

Guengerich FP (1992) Human cytochrome P-450 enzymes. Life Sci 50:1471-1478.

Heid CA, Stevens J, Livak KJ, and Williams PM (1996) Real time quantitative PCR. Genome Res 6:986-994.

Jackson JP, Ferguson SS, Moore R, Negishi M, and Goldstein JA (2004) The constitutive active/ androstane receptor regulates phenytoin induction of Cyp2c29. Mol Pharmacol 65:1397-1404.

Jackson JP, Ferguson SS, Negishi M, and Goldstein JA (2006) Phenytoin induction of the cyp2c37 gene is mediated by the constitutive androstane receptor. Drug Metab Dispos 34:2003-2010.

Kaminsky LS and Fasco MJ (1992) Small intestinal cytochromes P450. Crit Rev Toxicol 21: $407-422$.

King LM, Gainer JV, David GL, Dai D, Goldstein JA, Brown NJ, and Zeldin DC (2005) Single nucleotide polymorphisms in the CYP2J2 and CYP2C8 genes and the risk of hypertension. Pharmacogenet Genomics 15:7-13.

Konno Y, Kamino H, Moore R, Lih F, Tomer KB, Zeldin DC, Goldstein JA, and Negishi M (2010) The nuclear receptors constitutive active/androstane receptor and pregnane $\mathrm{x}$ receptor activate the Cyp2c55 gene in mouse liver. Drug Metab Dispos 38:1177-1182.

Kroetz DL and Zeldin DC (2002) Cytochrome P450 pathways of arachidonic acid metabolism Curr Opin Lipidol 13:273-283.

Larsen BT, Miura H, Hatoum OA, Campbell WB, Hammock BD, Zeldin DC, Falck JR, and Gutterman DD (2006) Epoxyeicosatrienoic and dihydroxyeicosatrienoic acids dilate human coronary arterioles via $\mathrm{BK}(\mathrm{Ca})$ channels: implications for soluble epoxide hydrolase inhibition. Am J Physiol Heart Circ Physiol 290:H491-H499.

Leemann TD, Transon C, Bonnabry P, and Dayer P (1993) A major role for cytochrome P450TB (CYP2C subfamily) in the actions of non-steroidal antiinflammatory drugs. Drugs Exp Clin Res 19:189-195.

Livak KJ and Schmittgen TD (2001) Analysis of relative gene expression data using real-time quantitative PCR and the 2(-Delta Delta C(T)) Method. Methods 25:402-408.

Löfgren S, Baldwin RM, Hiratsuka M, Lindqvist A, Carlberg A, Sim SC, Schülke M, Snait M, Edenro A, Fransson-Steen R, et al. (2008) Generation of mice transgenic for human CYP2C18 and CYP2C19: characterization of the sexually dimorphic gene and enzyme expression. Drug Metab Dispos 36:955-962.

Luo G, Zeldin DC, Blaisdell JA, Hodgson E, and Goldstein JA (1998) Cloning and expression of murine CYP2Cs and their ability to metabolize arachidonic acid. Arch Biochem Biophys 357:45-57. Nebert DW and Russell DW (2002) Clinical importance of the cytochromes P450. Lancet 360: $1155-1162$.

Nelson DR, Koymans L, Kamataki T, Stegeman JJ, Feyereisen R, Waxman DJ, Waterman MR, Gotoh O, Coon MJ, Estabrook RW, et al. (1996) P450 superfamily: update on new sequences, gene mapping, accession numbers and nomenclature. Pharmacogenetics 6:1-42.

Nelson DR, Zeldin DC, Hoffman SM, Maltais LJ, Wain HM, and Nebert DW (2004) Comparison of cytochrome P450 (CYP) genes from the mouse and human genomes, including nomenclature recommendations for genes, pseudogenes and alternative-splice variants. Pharmacogenetics 14: $1-18$.

Node K, Huo Y, Ruan X, Yang B, Spiecker M, Ley K, Zeldin DC, and Liao JK (1999) Antiinflammatory properties of cytochrome P450 epoxygenase-derived eicosanoids. Science 285: 1276-1279.

Node K, Ruan XL, Dai J, Yang SX, Graham L, Zeldin DC, and Liao JK (2001) Activation of Galpha $s$ mediates induction of tissue-type plasminogen activator gene transcription by epoxyeicosatrienoic acids. J Biol Chem 276:15983-15989.

Paine MF, Hart HL, Ludington SS, Haining RL, Rettie AE, and Zeldin DC (2006) The human intestinal cytochrome P450 "pie". Drug Metab Dispos 34:880-886.

Ponchel F, Toomes C, Bransfield K, Leong FT, Douglas SH, Field SL, Bell SM, Combaret V, Puisieux A, Mighell AJ, et al. (2003) Real-time PCR based on SYBR-Green I fluorescence: an alternative to the TaqMan assay for a relative quantification of gene rearrangements, gene amplifications and micro gene deletions. BMC Biotechnol 3:18. 
Scheer N, Kapelyukh Y, Chatham L, Rode A, Buechel S, and Wolf CR (2012) Generation and characterization of novel cytochrome P450 Cyp2c gene cluster knockout and CYP2C9 humanized mouse lines. Mol Pharmacol 82:1022-1029.

Seubert J, Yang B, Bradbury JA, Graves J, Degraff LM, Gabel S, Gooch R, Foley J, Newman J, Mao L, et al. (2004) Enhanced postischemic functional recovery in CYP2J2 transgenic hearts involves mitochondrial ATP-sensitive K+ channels and p42/p44 MAPK pathway. Circ Res $\mathbf{9 5}$. 506-514.

Seubert JM, Sinal CJ, Graves J, DeGraff LM, Bradbury JA, Lee CR, Goralski K, Carey MA, Luria A, Newman JW, et al. (2006) Role of soluble epoxide hydrolase in postischemic recovery of heart contractile function. Circ Res 99:442-450.

Tanaka E (1999) Gender-related differences in pharmacokinetics and their clinical significance. $J$ Clin Pharm Ther 24:339-346.

Tsao C-C, Foley J, Coulter SJ, Maronpot R, Zeldin DC, and Goldstein JA (2000) CYP2C40, a unique arachidonic acid 16-hydroxylase, is the major CYP2C in murine intestinal tract. Mol Pharmacol 58:279-287.

Tzveova R, Naydenova G, Yaneva T, Dimitrov G, Vandeva S, Matrozova Y, PendichevaDuhlenska D, Popov I, Beltheva O, Naydenov C, et al. (2015) Gender-specific effect of CYP2C8*3 on the risk of essential hypertension in Bulgarian Patients. Biochem Genet 53:319-333.

Veronese ME, Mackenzie PI, Doecke CJ, McManus ME, Miners JO, and Birkett DJ (1991)

Tolbutamide and phenytoin hydroxylations by cDNA-expressed human liver cytochrome P4502C9. Biochem Biophys Res Commun 175:1112-1118.
Wang H, Zhao Y, Bradbury JA, Graves JP, Foley J, Blaisdell JA, Goldstein JA, and Zeldin DC (2004) Cloning, expression, and characterization of three new mouse cytochrome p450 enzymes and partial characterization of their fatty acid oxidation activities. Mol Pharmacol 65:1148-1158

Wang Y, Wei X, Xiao X, Hui R, Card JW, Carey MA, Wang DW, and Zeldin DC (2005) Arachidonic acid epoxygenase metabolites stimulate endothelial cell growth and angiogenesis via mitogen-activated protein kinase and phosphatidylinositol 3-kinase/Akt signaling pathways. $J$ Pharmacol Exp Ther 314:522-532.

Waxman DJ and Azaroff L (1992) Phenobarbital induction of cytochrome P-450 gene expression. Biochem J 281:577-592.

Waxman DJ and Holloway MG (2009) Sex differences in the expression of hepatic drug metabolizing enzymes. Mol Pharmacol 76:215-228.

Zeldin DC, DuBois RN, Falck JR, and Capdevila JH (1995) Molecular cloning, expression and characterization of an endogenous human cytochrome P450 arachidonic acid epoxygenase isoform. Arch Biochem Biophys 322:76-86.

Address correspondence to: Dr. Darryl C. Zeldin, NIH/NIEHS, 111 T.W. Alexander Drive, Building 101, Room A214, Research Triangle Park, NC 27709. E-mail: zeldin@niehs.nih.gov 